\title{
Mechanism of Selective Inhibition of Yohimbine and Its Derivatives in Adrenoceptor $\alpha 2$ Subtypes
}

\author{
Liu Hai-Bo, ${ }^{1}$ Peng Yong, ${ }^{1}$ Huang Lu-qi, ${ }^{2}$ Xu Jun, ${ }^{3}$ and Xiao Pei-Gen ${ }^{1}$ \\ ${ }^{1}$ Institute of Medicinal Plant Development, Chinese Academy of Medical Sciences, Beijing 100193, China \\ ${ }^{2}$ Institute of Chinese Materia Medica, China Academy of Chinese Medical Sciences, Beijing 100700, China \\ ${ }^{3}$ School of Pharmaceutical Sciences, Sun Yat-sen University, Guangzhou 510006, China \\ Correspondence should be addressed to Peng Yong; ypeng@implad.ac.cn
}

Received 12 November 2012; Revised 22 March 2013; Accepted 2 April 2013

Academic Editor: Mehmet Emin Duru

Copyright (C) 2013 Liu Hai-Bo et al. This is an open access article distributed under the Creative Commons Attribution License, which permits unrestricted use, distribution, and reproduction in any medium, provided the original work is properly cited.

\begin{abstract}
Some natural alkaloids from medicinal plants, such as yohimbine and its derivatives, have been reported with adrenoceptor (AR) $\alpha 2$ subtypes inhibiting activity. In trying to address the possible mechanism of the action, a set of homology models of AR $\alpha 2$ was built based on MOE. After that, docking and molecular dynamics methods were used to investigate the binding modes of yohimbine and its 2 derivatives in the active pocket of adrenoceptor $\alpha 2$ subtype $\mathrm{A}, \mathrm{B}$, and C. The key interactions between the 3 ligands and the 3 receptors were mapped. Binding mode analysis presents a strong identity in the key residues in each subtype. Only a few differences play the key role in modulating selectivity of yohimbine and its derivatives. These results can guide the design of new selective AR $\alpha 2$ inhibitors.
\end{abstract}

\section{Introduction}

Adrenoceptor (AR) is a signal receptor of epinephrine and norepinephrine and is mainly distributed in myocardial, vascular, and nervous system. Till now, 9 AR subtypes have been reported. They are classified into subtypes $\alpha 1, \alpha 2$ and $\beta$ according to the pharmacological functions and sequences. $\alpha 1$ receptors have subtype $A, B$, and $D . \alpha 2$ receptors have subtypes $A, B$, and $C$. $\beta$ receptors have $\beta 1, \beta 2, \beta 3[1,2]$.

Since discovered in 1948, AR family has always been one of the most important targets for drug discovery. Both the agonist and the antagonist on different AR subtypes can be leading compounds with pharmacological effects, particularly the significant cardiovascular effect. Looking for the candidate with high selectivity among the AR subtypes is the emphasis in drug development. The $\alpha 2$ ARs are widely distributed throughout the peripheral and CNS. Agonists acting at $\alpha 2$ ARs have analgesic properties following supraspinal [3], spinal [4], peripheral [5], and systemic administration [6].

Some natural products acting on the AR receptor have been found as the active ingredients of traditional Chinese medicine. All the compounds are alkaloids and are the AR blockers. According to the skeleton, the compounds can be divided into three categories: rauwolfia alkaloids, such as yohimbine; protoberberine, such as berberine; and aporphine, such as xylopine (Table 1).

Yohimbine (CAS: 146-48-5) was first isolated from bark of a West African tree Pausinystalia yohimbe (Rubiaceae) in 1945. The plant has a long history of human use as a stimulant and aphrodisiac, especially as a herbal supplement to improve erectile function. To date, Yohimbine was also found in Alchornea floribunda Müll. Arg. (Euphorbiaceae) [14], Rauvolfia vomitoria, Rauwolfia nitida, Rauwolfia serpentine, Rauvolfia yunnanensis, and others [15].

The pharmacological research on Yohimbine has been started since 1949 [16]. The reported activities include antidiuretic, antiadrenaline, mydriatic, serotonin antagonist, and others. The most remarkable activity is selective inhibition on ARs, high affinity for the AR $\alpha 2$ subtypes, and moderate affinity for the $\alpha 1$ subtypes. Binding affinities among the AR $\alpha 2$ subtypes are $\alpha 2 \mathrm{C}(0.88 \mathrm{nM})>\alpha 2 \mathrm{~A}(1.4 \mathrm{nM})>\alpha 2 \mathrm{~B}$ $(7.1 \mathrm{nM})[13,17,18]$. Because of the high selectivity on AR subtypes, Yohimbine attracted more attention $[11,12]$. Lots of pharmacological data have been reported, while the mechanism of the action is still unclear. 
TABLE 1: The alkaloids with AR blocking activities.

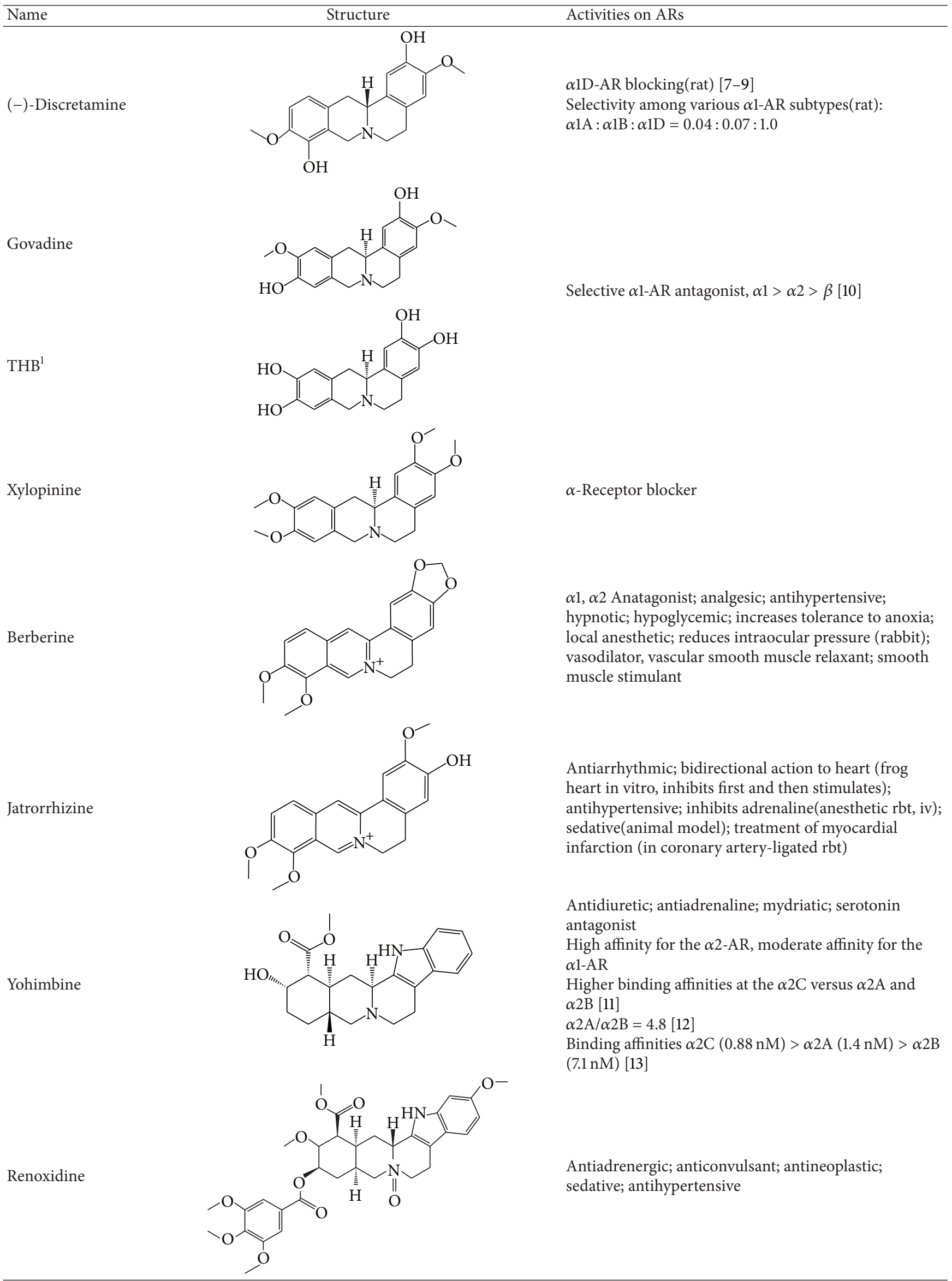


TABle 1: Continued.

Isocorydine Activities on ARs

${ }^{1}(+/-)-2,3,10,11-$ Tetrahydroxy tetrahydroprotoberberine HBr.

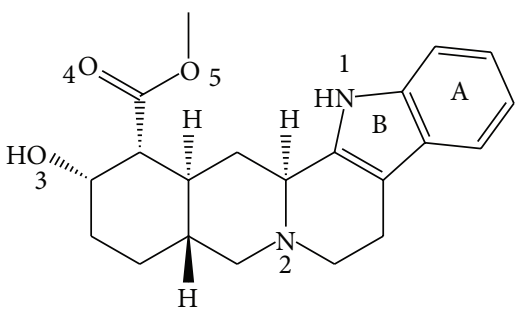

Yohimbine

(a)<smiles>COC(=O)CNC(=O)[C@H]1[C@@H](O)CC[C@H]2CN3CCc4c([nH]c5ccccc45)[C@H]3C[C@H]21</smiles>

Yohimbine monoglycine ester (YME)

(b)<smiles>NCCCNC(=O)[C@H]1[C@@H](O)CC[C@H]2CN3CCc4c([nH]c5ccccc45)[C@H]3C[C@H]21</smiles>

Yohimbine alkyl amine (YAA)

(c)

FIGURE 1: Yohimbine and its 2 derivatives.

In this paper, three-dimensional homology models were built for each AR $\alpha 2$ subtype. By using molecule stimulation method, the binding modes of Yohimbine, Yohimbine monoglycine ester (YME), and Yohimbine alkyl amine (YAA) were analyzed (Figure 1). The mechanism of selectivity and the key residues were investigated.

\section{Data and Methods}

2.1. Homo Modeling. The AR receptor belongs to heterotrimeric guanine nucleotide-binding protein coupled receptors (GPCRs). The proteins in GPCR superfamily usually have low sequence identities but with high structure similarities. The binding sites usually locate among the seven transmembrane (TM) helices; together with three extracellular loops. GPCR receptors play important roles in drug discovery [19]. Due to the hardness in the crystallization of GPCRs, homology modeling is a main approach for the structurebased drug design of GPCRs [20].

The secondary structure assignments are annotated with color-coded bars above the sequences. Helixes are in red, strands are in yellow, 1-4 turns are in blue, and 1-5 turns are in green. The conserved residues were marked with dark color.
To date, our understanding of GPCR structure is mainly based on the high-resolution crystal protein structure. Computational modeling has also predicted that GPCRs share a seven membrane-spanning a-helix topology as a common structural property. Likewise, mutagenesis studies have tentatively identified individual transmembrane domains with specific roles in signal transduction function that are conserved throughout the GPCR family. Since 2007, several Homo AR $\beta$-subtypes were reported [21-24]. Among the crystal data, $2 \mathrm{RH1}$, a complex of $\beta 2$ subtype with agonist carazolol, is an ideal template for the modeling of AR family $[25,26]$.

The alignment errors increase rapidly when the sequence identity of two proteins is less than 30\% [27]. For GPCR homology, the sequence identity is usually less than 25\% [20]. Therefore, automated homology modeling of GPCRs is likely to result in more errors [28]. The errors in alignments cannot be justified by energy optimizations, molecular dynamics, or distance geometry refinements. The alignments become critical for the quality of the homology model. Multiple sequence alignments are adopted in order to enhance the alignment quality. We have used $8 \mathrm{AR}$ subtypes in the sequence alignment. As shown in Figure 2, all the subtypes keep a high 

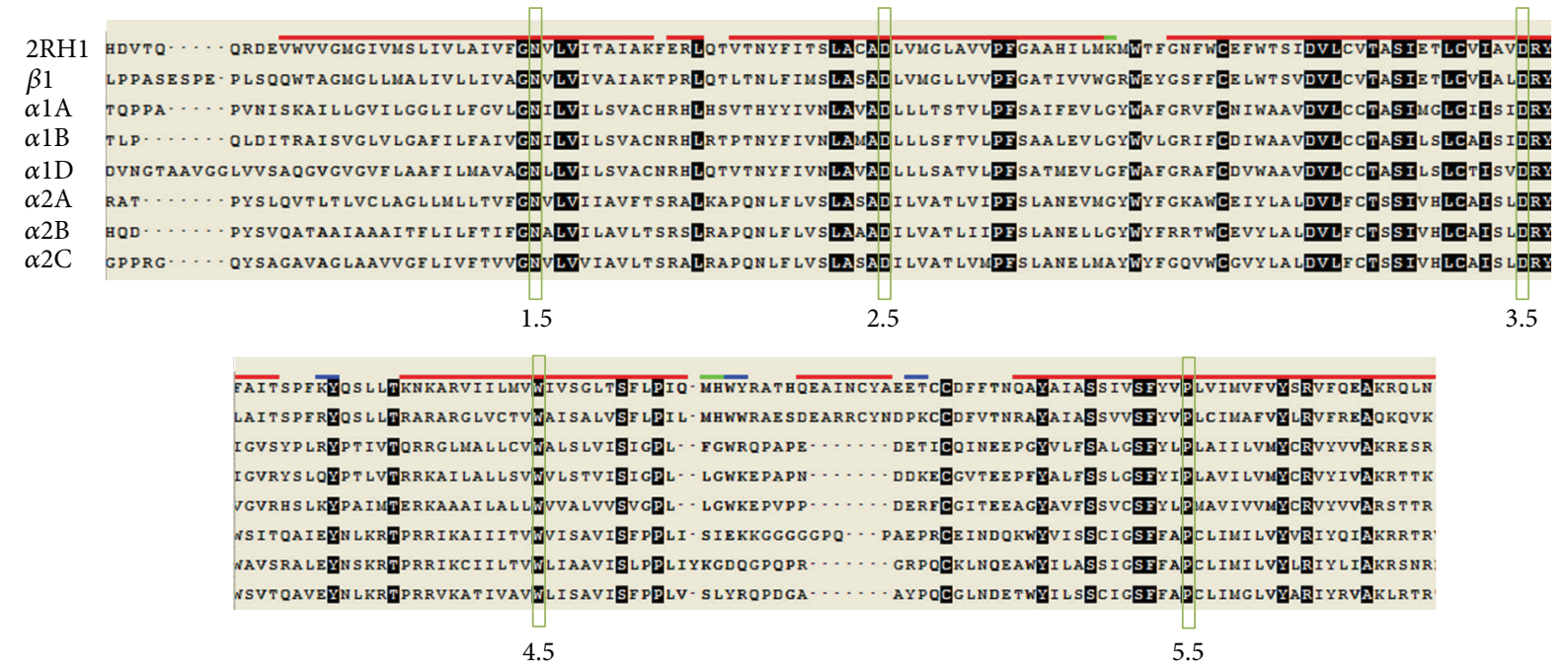

FIGURE 2: Alignment of the 8 AR subtypes.
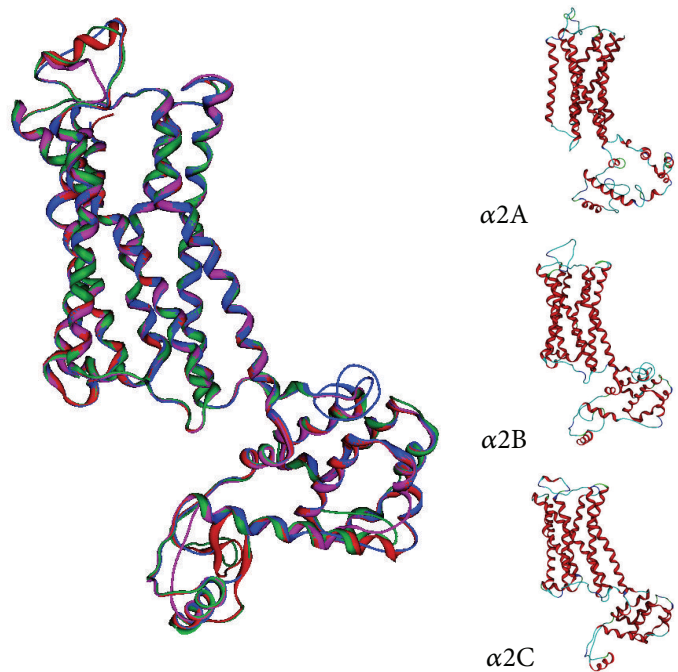

FIGURE 3: The homology models of three $\alpha 2$ subtypes. The left is the template superposed by the three homology models, 2RH1: red, $\alpha 2 \mathrm{~A}$ : green, $\alpha 2 \mathrm{~B}$ : blue, and $\alpha 2 \mathrm{C}$ : purple.

identity in the TM helices. For the binding site is exactly constituted by the TMs, the homo model may be of high reliability to estimate the interaction between the ligand and AR.

Homo models of AR $\alpha 2 \mathrm{~A}, \mathrm{~B}$ and $\mathrm{C}$ were constructed with the homology modeling module of the molecular operating environment (MOE) [29]. The AMBER99 force field was applied to the homology modeling. The carboxyl-terminal and amino-terminal modeling was disabled. The scoring method was GB/VI [30]. Ten models were built by MOE for each subtype, kept in a MOE database, and ranked by the GB/VI scores. The best model was kept for the following energy optimizing.

Partial charges for all atoms were calculated. Then, the energy was minimized by means of AMBER 99 force field, and the ending condition of the calculation was the root mean square (RMS) of the two conformer energies being less than $0.1 \mathrm{kcal} \mathrm{mol}^{-1} \AA^{-1}$. Furthermore, molecular mechanics (MD) simulations were applied, in MOE. The process consisted of two steps.

(1) The MD simulations were performed with all $\mathrm{C} \alpha$ atoms being fixed. The temperature was increased from $0 \mathrm{~K}$ to $300 \mathrm{~K}$ within $60 \mathrm{ps}$ (picoseconds); an NVT (constant volume/constant-temperature) molecular dynamic simulation of $100 \mathrm{ps}$ at $300 \mathrm{~K}$ was carried out with a time-step length of 0.002 ps.

(2) The atoms in the TM helixes were set flexible for conformation changes. The MD experiments simulated conformation changes in $1 \mathrm{~ns}$ timescale. The final model was obtained by energy minimization till an RMS of $0.05 \mathrm{kcal} \mathrm{mol}^{-1} \AA^{-1}$ was reached.

2.2. Docking. Docking experiments were carried out to explore the binding modes of three antagonists, Yohimbine, 

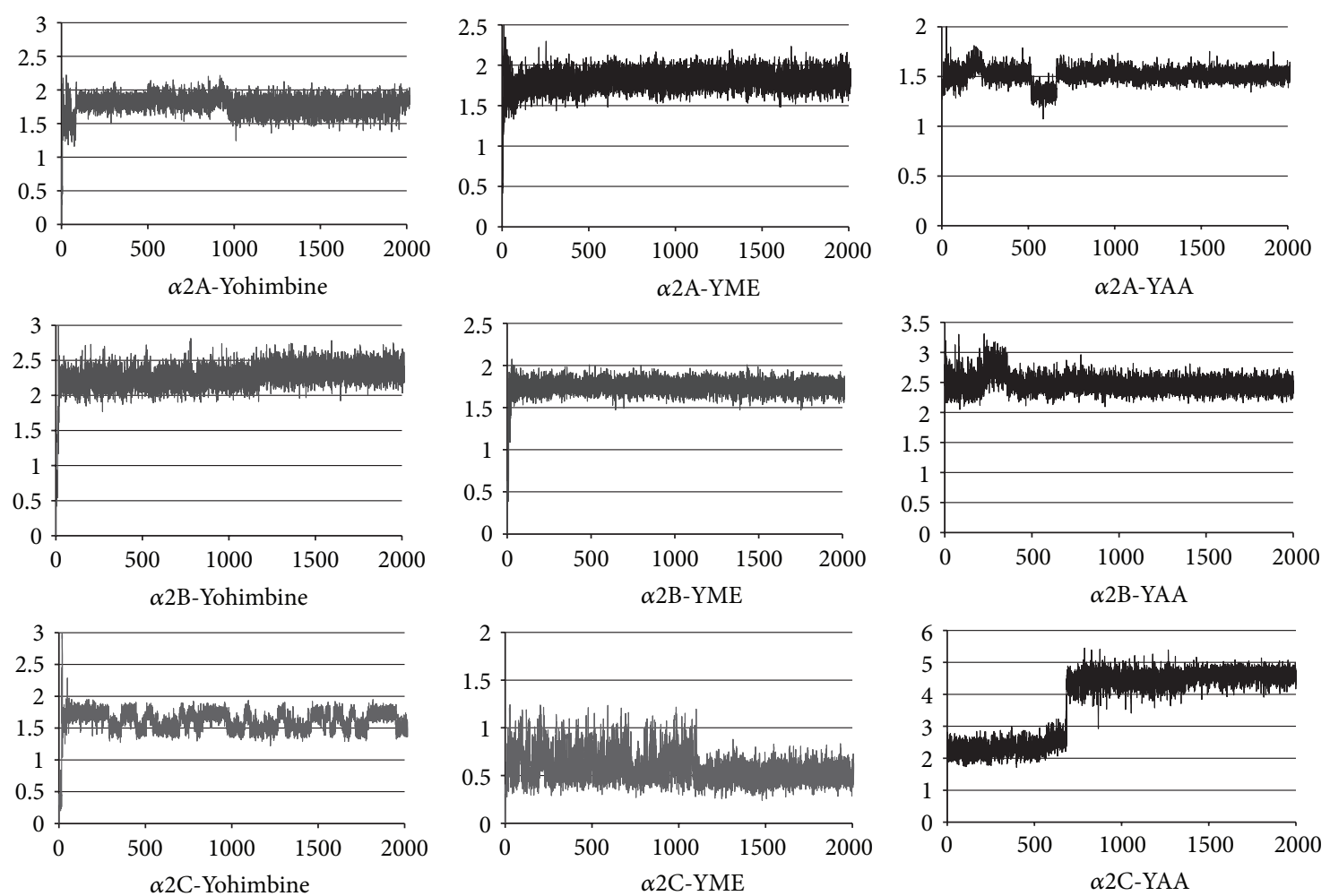

FIgURE 4: The RMSD fluctuation during MD simulation.

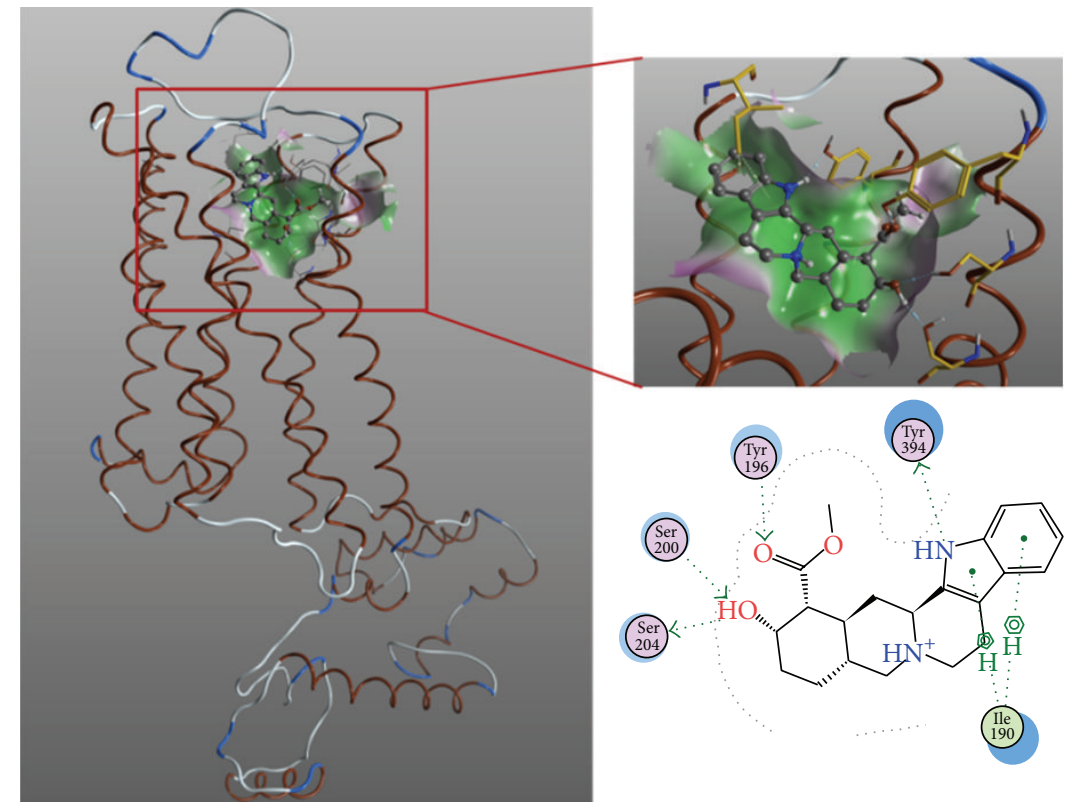

FIGURE 5: The binding mode of yohimbine with AR $\alpha 2 \mathrm{~A}$.

YME, and YAA. All the ligands were built in MOE Builder. Their three-dimensional structures were energy minimized by MOE using MMFF94x force field. The initial poses were assigned by Flexible Alignment module of MOE, with the agonist carazolol in $2 \mathrm{RH} 1$ as the template. The 3 ligands were docked into $3 \alpha 2$ subtypes, respectively. Totally 9 docking experiments were carried out.

Docking used the algorithm MOE Dock. The default settings were used. For each position, 700 iterations were generated to optimize the interactions at a location. A database 


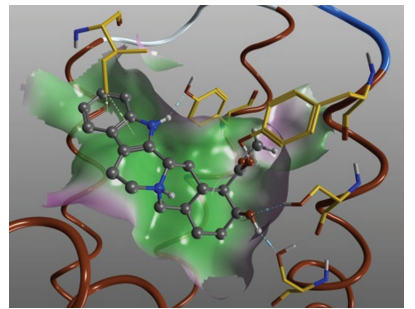

$\alpha 2 \mathrm{~A}-$ Yohimbine

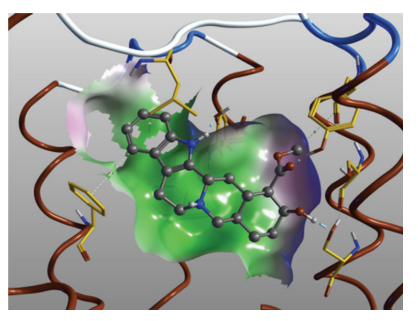

$\alpha 2 \mathrm{~B}$-Yohimbine

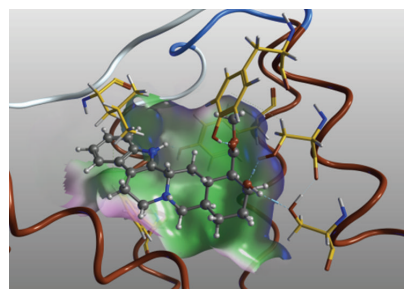

$\alpha 2$ C-Yohimbine

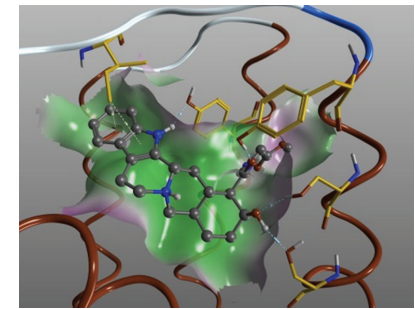

$\alpha 2 \mathrm{~A}-\mathrm{YME}$

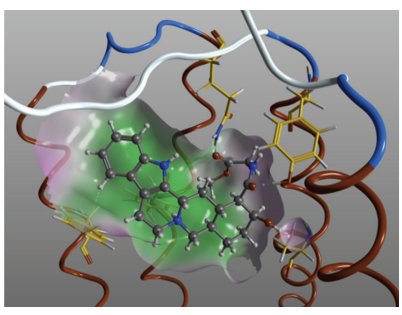

$\alpha 2 \mathrm{~B}-\mathrm{YME}$

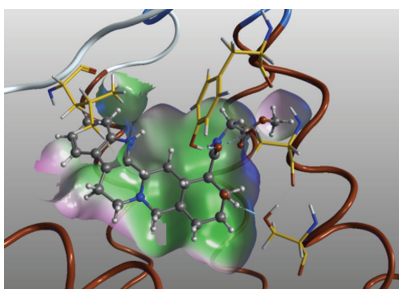

$\alpha 2 \mathrm{C}-\mathrm{YME}$

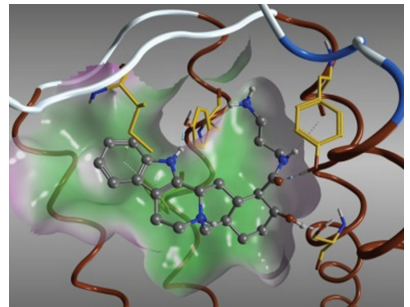

$\alpha 2 \mathrm{~A}-\mathrm{YAA}$

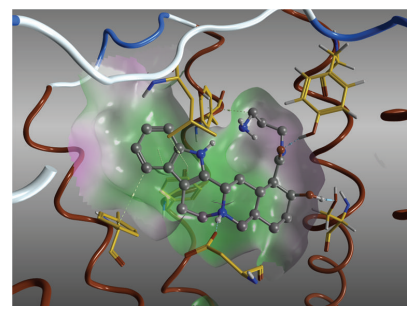

$\alpha 2 \mathrm{~B}-\mathrm{YAA}$

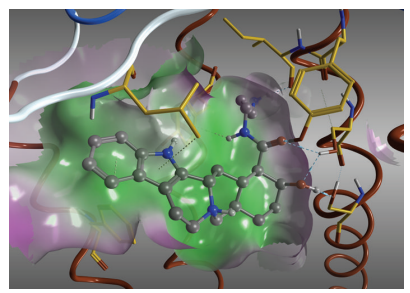

$\alpha 2 \mathrm{C}-\mathrm{YAA}$

FIgURE 6: The pose of 3 ligands in the binding pocket of 3 subtypes.

of 25 complexes for each receptor with each ligand was generated. MMFF94 force field was used. Interactive forces between the ligand and the receptor include hydrogen bonding, aromatic interactions, and hydrophobic interactions. Of these, hydrogen bonding is the strongest interaction followed by aromatic interactions and last hydrophobic interactions. Therefore, the best complex was judged to be the complex with the greatest number of hydrogen bonds to side chain atoms, aromatic interactions, and placement in the helical bundle. No explicit water molecules were added during docking simulation. Solvation and entropic effects were not taken into account either.

The top 3 reasonable binding modes were picked, based upon compatibility with the reported mutation results and binding data, as well as energetic considerations, from the 10 resulting receptor-ligand complexes. A further refinement was done in MOE. The residues within $6 \AA$ from the ligand were set to be flexible. Energy minimization was carried out by conjugated gradient minimization with the MMFF94x force field, until an RMSD of $0.1 \mathrm{kcal} \mathrm{mol}^{-1} \AA^{-1}$ was reached.

2.3. Dynamic Simulations on Docking Results. The top 3 complexes for each ligand and receptor were then subjected to two-stage molecular dynamics simulations. The simulation was performed for $1 \mathrm{fs}$ time step with $200 \mathrm{ps}$ of gradually heating phase from $0 \mathrm{~K}$ to $300 \mathrm{~K}$, followed by 2000 ps equilibrium (data collection) phase. The output databases contained
11000 entries collected during the equilibrium phase. The simulation used NVT parameters (holding constant moles, volume, and temperature) and calculated potential energy ( $U$ in $\left.\mathrm{kcal} \mathrm{mol}^{-1}\right)$, temperature ( $T$ in kelvins), pressure $(P$ in $\mathrm{Kpa})$, total energy $\left(E\right.$, kinetic and potential in $\left.\mathrm{kcal} \mathrm{mol}^{-1}\right)$, and enthalpy $\left(H, E+P V\right.$ in $\left.\mathrm{kcal} \mathrm{mol}^{-1}\right)$. The complex with the least RMSD fluctuation was considered as the best one.

\section{Result and Discussion}

Figure 3 shows the template protein $2 \mathrm{RH} 1$ and the three established homology models. There are little structural differences among the template and the models in the $7 \mathrm{TM}$ helices. The three-dimensional structure of binding pocket, surrounded by TM3, TM5, TM6, and eLP2, is especially close to each other among the 3 models. Because of the lack of conserved residues in loop domain, there are much more difference in the 3 endocellular loops and 3 extracellular loops. Since the active pocket is located in the upper half of the TMs, the differences of the loops do not affect the study on the binding mode of ligands. Therefore, the model can be used for the following molecular docking studies.

The 3 ligands have been docked to the homology structures of the 3 AR subtypes A, B, and C, respectively. For each combination, the most reasonable result was chosen as the start conformation for a further molecule dynamics optimization. The RMSD trajectories generated from the MD 


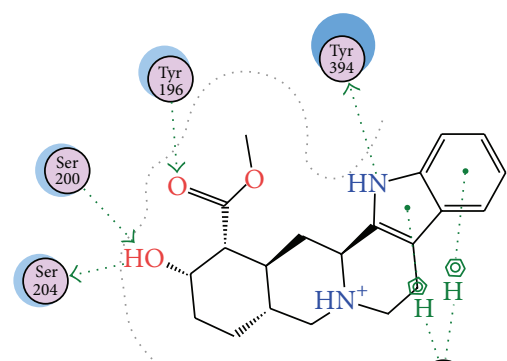

(iiis)

$\alpha 2$ A-Yohimbine

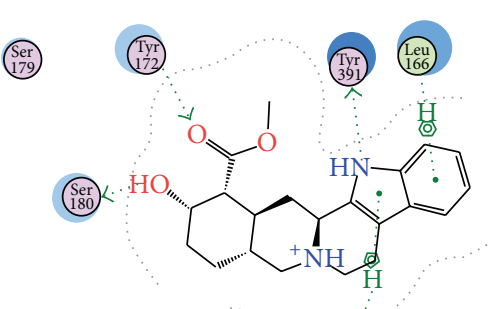

(38) (1820)

$\alpha 2 \mathrm{~B}$-Yohimbine

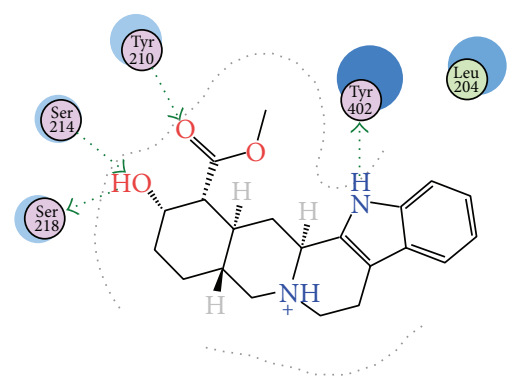

$\alpha 2 \mathrm{C}$-Yohimbine

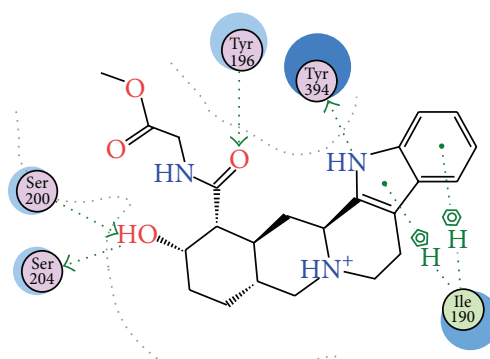

$\alpha 2 \mathrm{~A}-\mathrm{YME}$

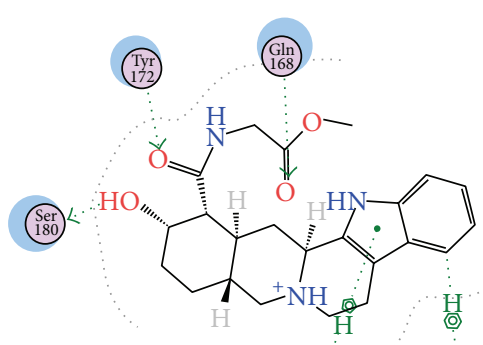

(iiiz)

$\alpha 2 \mathrm{~B}-\mathrm{YME}$

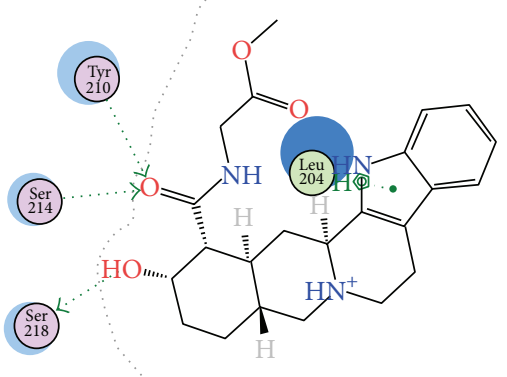

$\alpha 2 \mathrm{C}-\mathrm{YME}$

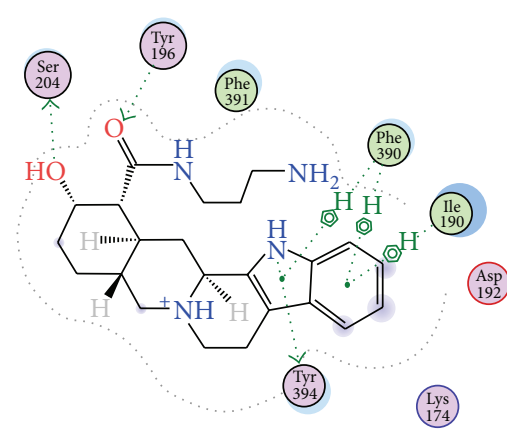

$\alpha 2 \mathrm{~A}-\mathrm{YAA}$

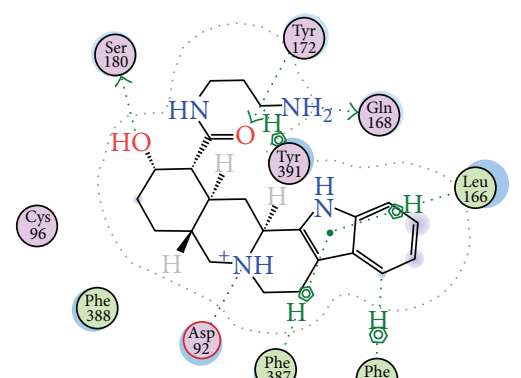

$\alpha 2 \mathrm{~B}-\mathrm{YAA}$

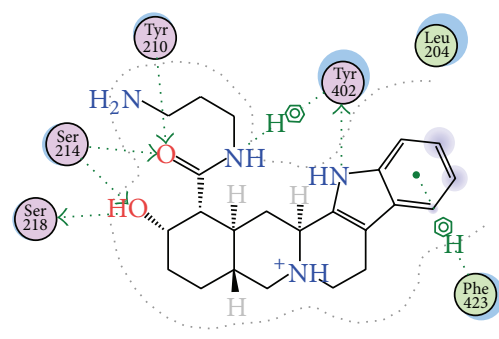

$\alpha 2 \mathrm{C}-\mathrm{YAA}$

FIGURE 7: The binding modes of the 3 antagonists to 3 subtypes. Blue arrows represent hydrogen bonds formed with backbone atoms; green arrows represent hydrogen bonds formed with side-chain atoms.

simulation were shown in Figure 4. The final model was obtained by averaging the structures from the 1.5 2 ns trajectories of MD simulations. The model energy was minimized till an RMS of $0.05 \mathrm{kcal} \mathrm{mol}^{-1} \AA^{-1}$ was reached.

Figure 5 shows the binding mode of the Yohimbine in the $\alpha 2 \mathrm{~A}$ three-dimensional structure. The 3 ligands' 9 binding modes are depicted in Figures 6 and 7. The three-dimensional views are listed in Figure 6 and the ligand interaction maps are listed in the Figure 7. All the antagonists have the same orientation. The hydrophobic ends point to the opening of the pocket, and interact, through arene- $\mathrm{H}$ interaction, with Phe, Leu, or Ile residues on TM6 and eLP3. The polar ends face the bottom of the pocket; hydrogen bonds are formed with Tyr5.38, Ser5.42, and Ser5.46. The "hot" residues were identified by summarizing the interactions between the 3 ligands and the $3 \alpha 2$ subtypes by $2 \mathrm{D}$ contact diagram of $\mathrm{MOE}$ (Figure 7, Table 2).
Table 2 summarized the interaction, hotspot residues, binding free energy, and reported $p \mathrm{Ki}$ [11]. As can be seen, the binding mode of yohimbine is very close to that of YME and YAA. The interaction between the 3 ligands and the AR2 receptors mainly includes 4 aspects: (a) hydrogen bonds between oxygen atom 3 and 4 with polar residues Y5.38 and S5.46; (b) nitrogen atom 1 as a hydrogen bond donor formed a hydrogen bond with Y6.55; (c) ring A and the ring B contact with the aromatic/aliphatic residues, such as F6.51, I/L5.32, by arene-H interaction; (d) S5.42 may account for the selective inhibition of yohimbine and its derivatives.

As common structural characteristics through the three AR $\alpha 2$ subtypes, Y5.38, S5.42, S5.46, I/L5.32, and F6.51 are "hot" residues, the most interactive with the ligands (Table 2). The hydrogen bond interactions are the main contributors to binding affinities, which mainly located at the bottom of the AR binding pocket. The aromatic/aliphatic interactions 
TABle 2: Summary on the interactions between 3 ligands and the $3 \alpha 2$ subtypes.

\begin{tabular}{|c|c|c|c|c|c|c|c|c|c|c|}
\hline \multirow{2}{*}{ ligands } & \multirow{2}{*}{ ARs } & \multicolumn{5}{|c|}{ Hydrogen bond } & \multicolumn{2}{|c|}{ Arene-H/arene-arene } & \multirow{2}{*}{$E\left(\mathrm{~kJ} \cdot \mathrm{mol}^{-1}\right)$} & \multirow{2}{*}{$p \mathrm{Ki}(\mu \mathrm{M})$} \\
\hline & & Y5.38 & S5.42 & S5.46 & Y6.55 & Others & F6.51 & Others & & \\
\hline Yoh. & $\alpha 2 \mathrm{~A}$ & $\mathrm{O}-4$ & $\mathrm{O}-3 / 4$ & $\mathrm{O}-3$ & $\mathrm{~N}-1$ & & & Ring-A/B-I5.32 & -80.95 & 8.75 \\
\hline Yoh. & $\alpha 2 \mathrm{~B}$ & $\mathrm{O}-4$ & & $\mathrm{O}-3$ & $\mathrm{~N}-1$ & & Ring-B & Ring-A-L5.32 & -61.37 & 8.14 \\
\hline Yoh. & $\alpha 2 \mathrm{C}$ & O-4 & $\mathrm{O}-3 / 4$ & $\mathrm{O}-3$ & N-1 & & & Ring-A/B-L5.32 & -94.06 & 9.01 \\
\hline YME & $\alpha 2 \mathrm{~A}$ & $\mathrm{O}-4$ & & $\mathrm{O}-3$ & $\mathrm{~N}-1$ & & Ring-B & Ring-A-I5.32 & -83.16 & 7.47 \\
\hline YME & $\alpha 2 \mathrm{~B}$ & $\mathrm{O}-4$ & & $\mathrm{O}-3$ & & & Ring-B & Ring-A/B-F412 & -81.11 & 7.44 \\
\hline YME & $\alpha 2 \mathrm{C}$ & $\mathrm{O}-4$ & $\mathrm{O}-3 / 4$ & $\mathrm{O}-3$ & & & Ring-B & Ring-A/B-L5.32 & -85.24 & 8.07 \\
\hline YAA & $\alpha 2 \mathrm{~A}$ & $\mathrm{O}-4$ & & $\mathrm{O}-3$ & $\mathrm{~N}-1$ & & Ring-A/B & Ring-A-I5.32 & -77.26 & 6.64 \\
\hline YAA & $\alpha 2 \mathrm{~B}$ & O-4 & & $\mathrm{O}-3$ & & NH2-D3.32 & Ring-A/B & Ring-A-L5.32 & -75.50 & 5.64 \\
\hline YAA & $\alpha 2 \mathrm{C}$ & $\mathrm{O}-4$ & $\mathrm{O}-3 / 4$ & $\mathrm{O}-3$ & & & Ring-A & Ring-B-L5.32 & -79.29 & 7.50 \\
\hline
\end{tabular}

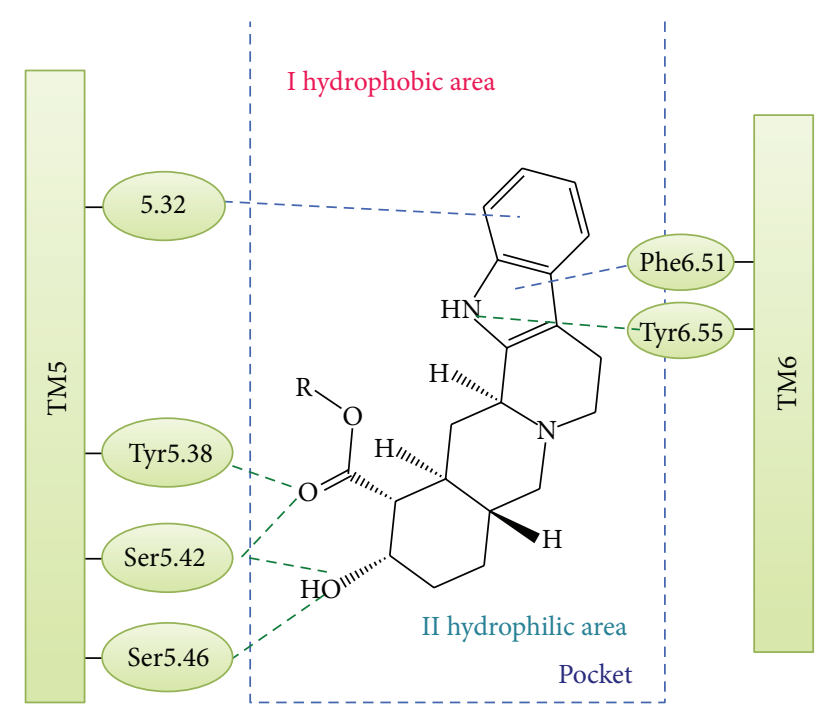

Figure 8: The 2 compartments binding modes for yohimbine and its derivatives.

are located at the opening of the pocket, close to the solvent. This area, the ligand tends to form arene- $\mathrm{H}$ bindings and hydrophobic interactions with "hot" residues, such as F6.51 and I/L5.32. Therefore, a good ligand should have at least one hydroxyl group at one end and two aromatic rings at the other end.

The root of the selectivity of the yohimbine and its derivatives may be the hydrogen bonds on S5.42. As shown in Table 2, the complexes with a hydrogen bond on S5.42 usually have a relatively higher $p \mathrm{Ki}$ value. That also presents an explanation on yohimbine higher selectivity on $\mathrm{AR} \alpha 2 \mathrm{C}$.

All the active pockets of AR $\alpha 2$ were formed by TM3, TM5, TM6, TM7, and eLP2. The pocket can be divided into two compartments (Figure 8). Compartment I is hydrophobic and close to the entrance of the pocket. Most residues in compartment I are aromatic/aliphatic, such as Phe, Leu, Ile and others. The most important of them are Phe6.51 and the Ile190 ( $\alpha 2 \mathrm{~A}, \mathrm{eLP} 2)$, Leu166 ( $\alpha 2 \mathrm{~B}, \mathrm{eLP} 2)$, and Leu204 ( $\alpha 2 \mathrm{C}$, eLP2). Compartment II is a hydrophilic bottom of the binding pocket and has more hydrogen bonding opportunities, hosting "hot" residues as well: Tyr5.38, Ser5.42 and Ser5.46 on TM5.

Consequently, as to the structural characteristics of yohimbine and its derivatives, the 3 and 4 oxygen atoms and $\mathrm{A}, \mathrm{B}$ rings (Figure 1) play the key role in the contact with ARs. The two oxygen atoms form "W" type 4 hydrogen bonds with Tyr5.38, Ser5.42, and Ser5.46, which all locate on TM5. The hydrogen bonds on Tyr5.38 and Ser5.46 are relatively stronger than those on Ser5.42. The two nitrogen atoms in the scaffold of yohimbine also interact with the residues on TM6 in some situations more or less. No. 1 nitrogen atom usually forms hydrogen bond with Tyr6.55; thus, it contributes more for the three ligands' affinity on AR $\alpha 2$. The hydrogen bonds between O-3 and S5.42 may contribute more in the formation of the selectivity of the ligands.

\section{Conclusions}

A set of homology models was built for AR $\alpha 2$ subtypes, based on multisequence alignments and secondary structure analyses. The 3 ligands were docked into 3 AR $\alpha 2$ subtype models. Molecular dynamics was carried to find the stable binding mode. The "hot" residues and the mechanism of the action are identified by comparing the 9 ligand-receptor complexes. These discoveries can be important for new AR $\alpha 2$ selective antagonist design.

\section{Acknowledgments}

The authors would like to thank Dr. Weiyu Lin for assistance in MOE SVL programing. The work in this paper was supported by the Ministry of Science and Technology of China, Special Project SB2007FY020.

\section{References}

[1] M. T. Piascik, E. E. Soltis, M. M. Piascik, and L. B. Macmillan, " $\alpha$-adrenoceptors and vascular regulation: molecular, pharmacologic and clinical correlates," Pharmacology and Therapeutics, vol. 72, no. 3, pp. 215-241, 1996.

[2] B. Rozec and C. Gauthier, " $\beta 3$-Adrenoceptors in the cardiovascular system: putative roles in human pathologies," Pharmacology and Therapeutics, vol. 111, no. 3, pp. 652-673, 2006.

[3] T. Z. Guo, J. Y. Jiang, A. E. Buttermann, and M. Maze, "Dexmedetomidine injection into the locus ceruleus produces 
antinociception," Anesthesiology, vol. 84, no. 4, pp. 873-881, 1996.

[4] S. V. R. Reddy and T. L. Yaksh, "Spinal noradrenergic terminal system mediates antinociception," Brain Research, vol. 189, no. 2, pp. 391-401, 1980.

[5] K. D. Davis, R. D. Treede, S. N. Raja, R. A. Meyer, and J. N. Campbell, "Topical application of clonidine relieves hyperalgesia in patients with sympathetically maintained pain," Pain, vol. 47, no. 3, pp. 309-317, 1991.

[6] L. Paalzow, "Analgesia produced by clonidine in mice and rats," Journal of Pharmacy and Pharmacology, vol. 26, no. 5, pp. 361363, 1974.

[7] F. N. Ko, J. H. Guh, S. M. Yu, Y. S. Hou, Y. C. Wu, and C. M. Teng, "(-)-Discretamine, a selective $\alpha(1 \mathrm{D})$-adrenoceptor antagonist, isolated from Fissistigma glaucescens," The British Journal of Pharmacology, vol. 112, no. 4, pp. 1174-1180, 1994.

[8] F. N. Ko, S. M. Yu, M. J. Su, Y. C. Wu, and C. M. Teng, "Pharmacological activity of (-)-discretamine, a novel vascular $\alpha$-adrenoceptor and 5-hydroxytrypamine receptor antagonist, isolated from Fissistigma glaucescens," The British Journal of Pharmacology, vol. 110, no. 2, pp. 882-888, 1993.

[9] A. Carrieri, N. B. Centeno, J. Rodrigo, F. Sanz, and A. Carotti, "Theoretical evidence of a salt bridge disruption as the initiating process for the $\alpha$ ld-adrenergic receptor activation: a molecular dynamics and docking study," Proteins, vol. 43, no. 4, pp. 382394, 2001.

[10] F. N. Ko, Y. L. Chang, C. M. Chen, and C. M. Teng, “( \pm$)$-govadine and $( \pm)$-THP, two tetrahydroprotoberberine alkaloids, as selective $\alpha 1$-adrenoceptor antagonists in vascular smooth muscle cells," Journal of Pharmacy and Pharmacology, vol. 48, no. 6, pp. 629-634, 1996.

[11] S. A. Bavadekar, G. Ma, S. M. Mustafa, B. M. Moore, D. D. Miller, and D. R. Feller, "Tethered yohimbine analogs as selective human $\alpha 2$ Cadrenergic receptor ligands," Journal of Pharmacology and Experimental Therapeutics, vol. 319, no. 2, pp. 739-748, 2006.

[12] W. Zheng, L. Lei, S. Lalchandani, G. Sun, D. R. Feller, and D. D. Miller, "Yohimbine dimers exhibiting binding selectivities for human $\alpha(2 \mathrm{a})$ - versus $\alpha(2 \mathrm{~b})$-adrenergic receptors," Bioorganic and Medicinal Chemistry Letters, vol. 10, no. 7, pp. 627-630, 2000.

[13] S. M. Mustafa, S. A. Bavadekar, G. Ma, B. M. Moore, D. R. Feller, and D. D. Miller, "Synthesis and biological studies of yohimbine derivatives on human $\alpha 2 \mathrm{C}$-adrenergic receptors," Bioorganic and Medicinal Chemistry Letters, vol. 15, no. 11, pp. 2758-2760, 2005.

[14] R. Paris and R. Goutarel, "African species of Alchornea; the presence of yohimbine in Alchornea floribunda (Euphorbiaceae).," Annales pharmaceutiques françaises, vol. 16, no. 1, pp. 15-20, 1958.

[15] J. J. Zhou, G. R. Xie, and X. J. Yan, Chemical Components of Source Plants in Traditional Chinese Medicine, Science Press, Beijing, China, 2009.

[16] C. M. Mac, C. I. Mc, and F. H. Shaw, "The action of yohimbine on excitation and propagation in nerve," The Australian Journal of Experimental Biology and Medical Science, vol. 27, part 1, pp. 115-122, 1949.

[17] M. J. Millan, A. Newman-Tancredi, V. Audinot et al., "Agonist and antagonist actions of yohimbine as compared to fluparoxan at alpha(2)-adrenergic receptors (AR)s, serotonin (5-HT)(1A), 5-HT(1B), 5-HT(1D) and dopamine $\mathrm{D}(2)$ and $\mathrm{D}(3)$ receptors.
Significance for the modulation of frontocortical monoaminergic transmission and depressive states," Synapse, vol. 35 , no. 2 , pp. 79-95, 2000.

[18] D. D. Schwartz and T. P. Clark, "Selectivity of atipamezole, yohimbine and tolazoline for alpha-2 adrenergic receptor subtypes: implications for clinical reversal of alpha- 2 adrenergic receptor mediated sedation in sheep," Journal of Veterinary Pharmacology and Therapeutics, vol. 21, no. 5, pp. 342-347, 1998.

[19] J. Saunders, "G-protein-coupled receptors in drug discovery," Bioorganic \& Medicinal Chemistry Letters, vol. 15, article 3653, 2005.

[20] R. Heilker, M. Wolff, C. S. Tautermann, and M. Bieler, "Gprotein-coupled receptor-focused drug discovery using a target class platform approach," Drug Discovery Today, vol. 14, no. 5-6, pp. 231-240, 2009.

[21] T. Warne, M. J. Serrano-Vega, J. G. Baker et al., "Structure of a $\beta 1$-adrenergic G-protein-coupled receptor," Nature, vol. 454, no. 7203, pp. 486-491, 2008.

[22] S. G. F. Rasmussen, H. J. Choi, D. M. Rosenbaum et al., "Crystal structure of the human $\beta 2$ adrenergic G-protein-coupled receptor," Nature, vol. 450, no. 7168, pp. 383-387, 2007.

[23] M. A. Hanson, V. Cherezov, M. T. Griffith et al., "A specific cholesterol binding site is established by the $2.8 \mathrm{~A}$ structure of the human beta2-adrenergic receptor," Structure, vol. 16, no. 6, pp. 897-905, 2008.

[24] M. P. Bokoch, Y. Zou, S. G. F. Rasmussen et al., "Ligand-specific regulation of the extracellular surface of a G-protein-coupled receptor," Nature, vol. 463, no. 7277, pp. 108-112, 2010.

[25] L. Ostopovici-Halip, R. Curpân, M. Mracec, and C. G. Bologa, "Structural determinants of the alpha2 adrenoceptor subtype selectivity," Journal of Molecular Graphics and Modelling, vol. 29, no. 8, pp. 1030-1038, 2011.

[26] V. Cherezov, D. M. Rosenbaum, M. A. Hanson et al., "Highresolution crystal structure of an engineered human $\beta 2$ adrenergic G protein-coupled receptor," Science, vol. 318, no. 5854, pp. 1258-1265, 2007.

[27] D. Baker and A. Sali, "Protein structure prediction and structural genomics," Science, vol. 294, no. 5540, pp. 93-96, 2001.

[28] I. D. Pogozheva, M. J. Przydzial, and H. I. Mosberg, "Homology modeling of opioid receptor-ligand complexes using experimental constraints," AAPS Journal, vol. 7, no. 2, pp. E434-E448, 2005.

[29] "Molecular Operating Environment in, Chemical Computing Group Inc," Sherbrooke Street West, Suite 910, Montreal, H3A 2R7, Canada, 2007.

[30] P. Labute, "The generalized born/volume integral implicit solvent model: estimation of the free energy of hydration using London dispersion instead of atomic surface area," Journal of Computational Chemistry, vol. 29, no. 10, pp. 1693-1698, 2008. 

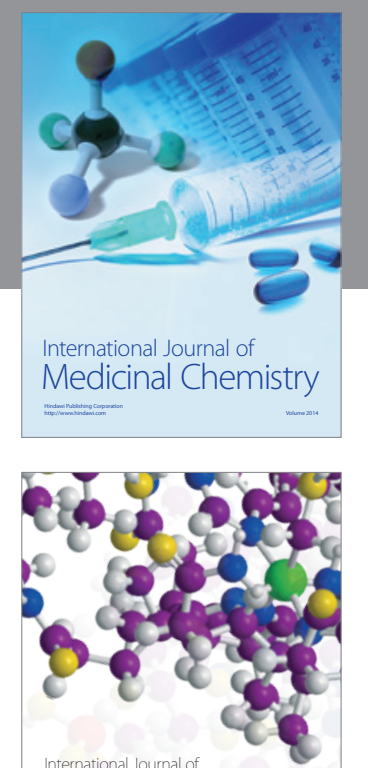

\section{Carbohydrate} Chemistry

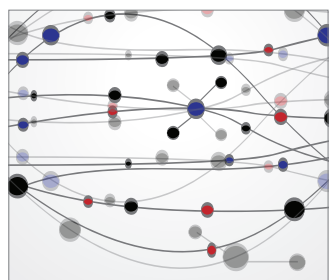

The Scientific World Journal
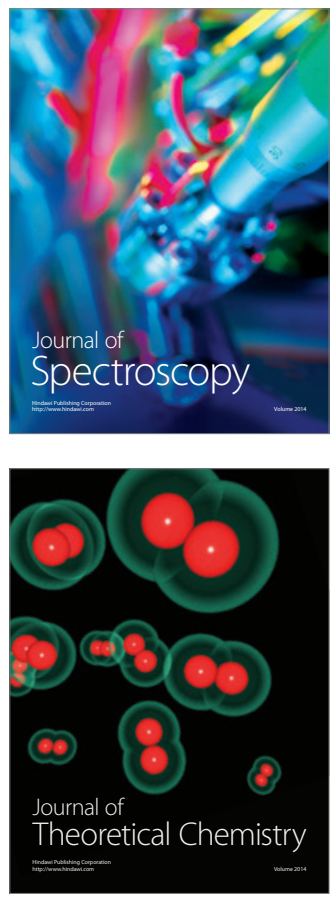
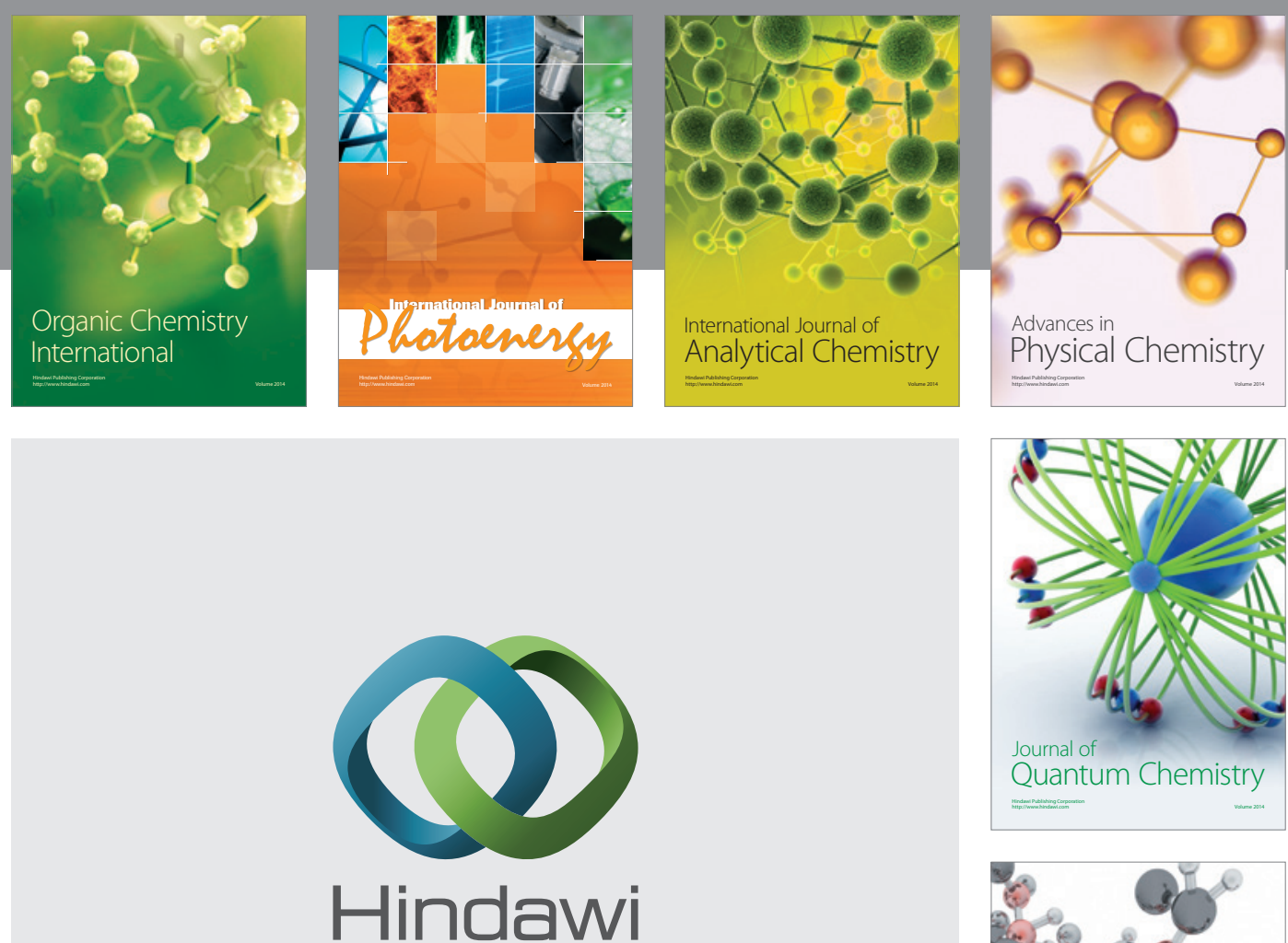

Submit your manuscripts at

http://www.hindawi.com

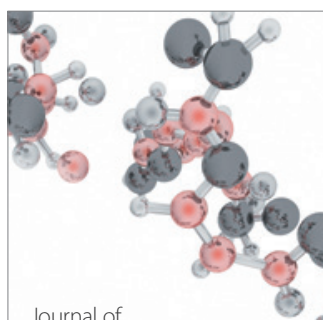

Analytical Methods

in Chemistry

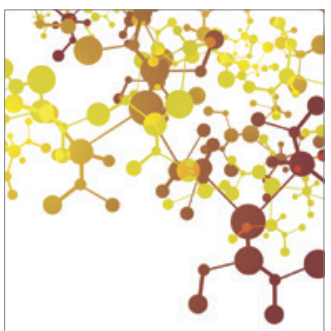

Journal of

Applied Chemistry

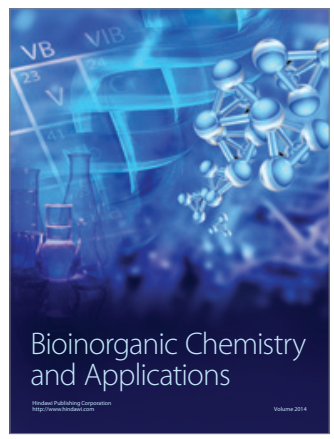

Inorganic Chemistry
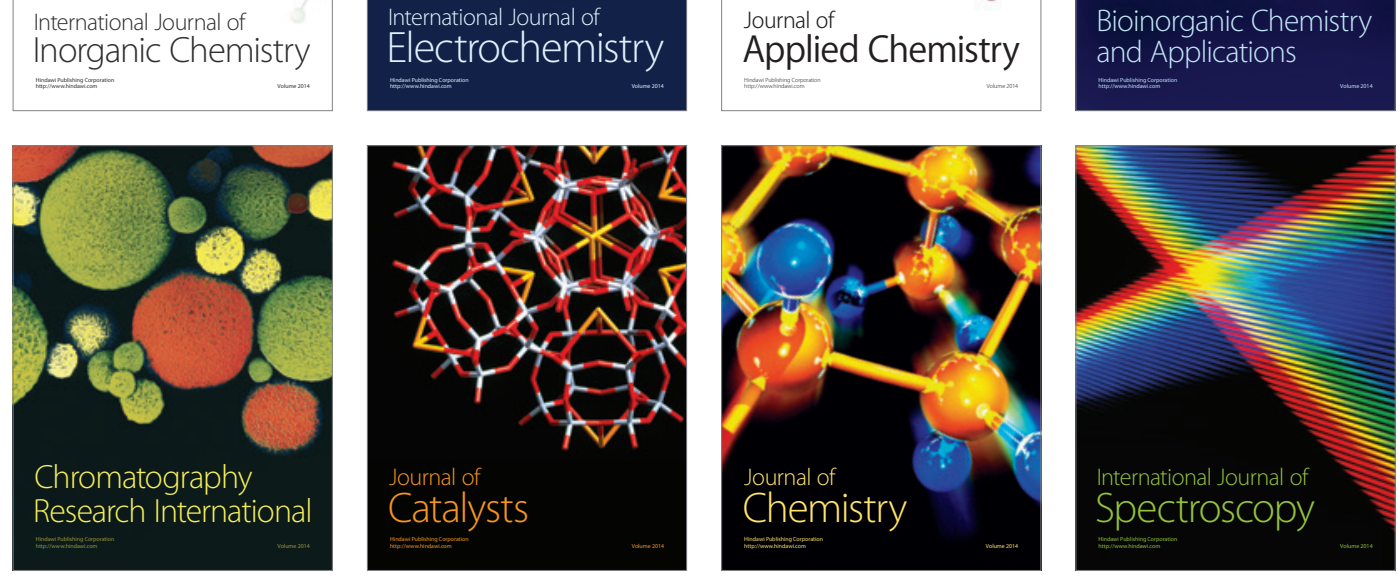